Monatsschrift f. Geburtshülfe u. Gynäkologie 1921;54:I-VI

\title{
Contents, Vol. 54, 1921
}

Inhalts - Verzeichnis.

Originalarbeiten.

Seit $1 / 8$

Abernetty, Carl, Die wichtigsten klinisch $\beta n$ Erschei-

nungen in den ersten fünf Wochenbettstagen bei Erst-

und Mehrgebärenden . 227

Ben thin, W. , Strahlentherapeutische Einzelbeobach-

tungen 34

Boettger, Max, Ein Beitrag zum verhornenden Platten-

epithelkarzinom des Ovariums

Brenner, M., Ein weiterer Fall von künstlicher Scheiden-

bildung nach Mori 112

Dyroff, Ein Fall von Scheidenwandabriß 7

Eisenstädter, David, Karzinomatöse Dermoidzysten des

Ovariums. (Hierzu Tafel III) 360

Fellner, Otfried 0., Über die Tätigkeit des Ovarium in der Schwangerschaft (interstitielle Zellen). (Hierzu

Tafel I-II) 88

Geller, Fr. Chr., Zur Ätiologie der Embryome . . . 352

Guggisberg,Hans, Die Wehensubstanzen in der Plazenta 277

Heimann, Fritz, Myom und Schwangerschaft .... 292

Hellmuth,Karl, Untersuchungen überBilirubinämiebeim

Neugeborenen, zugleich ein Beitrag zur Genese des

Icterus neonatorum 341

Herrmann, Edm. , Der Einfluß eines Corpus luteum- resp.

Plazentar-Lipoids auf Blutungen, menstruellen Zyklus

und Ausfallserscheinungen 152

Hieß, Viktor, Über die Grenzen der Leistungsfähigkeit

unserer heutigen Präviatherapie 165

Hinterstoißer, H., Ein Lithopädion 109

Hochenbichler,Ad., Über die Spaltrichtung der Eihäute

und die Bedeutung der Fasernrichtung der letzteren für

die Ausstoßung der Nachgeburt 143

Hürzeler, 0., Beitrag zur Frage der Beeinflussung des

Blutzuckers durch das Ovarium 215 ' $v-$

$\overline{\text { Seit }} \mathrm{3} / 8$

Sammelberichte. 
Hie geburtshülfliehe und gynäkologische Literatur in Finnland 1919. Von Priv.-Doz. Dr. Ed v. A. Björkenhei in

in Helsingfors . $\quad 318$

Bericht über die 1918-1919 in schwedischer Sprache er-

schienenen geburtshül Пich-gynäkologischen Arbeiten.

Von Dr. W. Brim km an in Lund 372

Aus den Grenzgebieten.

Der heutige Stand der tuberkulösen Peritonitis. Von Dr.

P. Wienecke in Berlin .. - 247

Die Rheinische Provinzial-Hebammenlehranstalt im Jahre

1920. Berichtet von Ed. Martin 259

Vereîns- und Literaturbeilage.

Originalsitzungsberichte aus geburtshülflich-gynäkologischen Gesellschaften. Gesellschaft für Geburtshülfe und Gynäkologie in Berlin. Sitzung von 12. XT. J920 .... 45

Sitzung vom 20. XII. 1920200

Sitzung vom 14. und 28. I. 1921263

Sitzung vom 11. II. 1921385

Gynäkologische Gesellschaft in Breslau. 61. und 62. Sitzung

vom 16: XI. und 14. XII. $1920 \quad 321$

Niederrhein.-westfälische Gesellschaft für Gynäkologie und

Geburtshülfe. 61. Sitzung vom 19. VI. 1920 ... . 46

62. Sitzung vom 6. XI. 192057

63. Sitzung vom 5. II. 1921377

Nordwestdeutsche Gesellschaft für Gynäkologie und Hamburger Geburtshülfliche Gesellschaft in Hamburg.

Sitzung vom 2. X. $1920 \quad 64$

Geburtshülflich-gynäkologische Gesellschaft in Wien. Sit

zung vom 1. VI., 15. VI. und 6. VII. 1920 ..... 73

12. Versammlung des „Nordisk Kirurgisk Forening” in

Christiania 3.-5. VII. 1919. Von Dr. V. Albeck

in Aarhus 117

Leopold Landau $\dagger \quad 127$

Wilhelm von Waldeyer-Hartz $\dagger \quad 198$

$-\mathrm{IV}-$

Seit $3 / 8$

J as dike, Rud. Th. von, Die fur die Verhütung der post-

operativen Thrombose und Embolie maßgebenden

Faktoren 1

Katz,Heinrich, Uber unvollstäudige äußere Uterusruptur 95 Kayser, Konr.,

Kohabitationsverletzung des hinteren

Scheidengewölbes im Puerperium 13

Lie be, W., Echte Eierstocksschwangerschaft 102

Liegner, B., Primäraffekt an der Portio 298

Lönne, Friedr., Über ältere und neuere Therapie der

Plazenta praevia unter besonderer Berücksichtigung der

abdominellen Schnittentbindung 190 
Martin, Ed., Plazenta-Opton als Wehenmittel .... 288 Mauthner, Ernst, Das Verhalten des Kapillarsystems bei

- der zyklischen Wandlung dér Uterusmukosa .... 81 Üttow, B. , Über eugenetische

Konzeptionsverhinderung

und die biologischen Grundlagen generativer Prophy-

laxe durchVasectomie und Autotransplantation der Hoden 219 Peffer, Josef, Die Erfolge der

Interposito uteri bei

Prolapsen 230

Puppel, Ernst, Die therapeutische Verwertung der

Plazenta 280

Richter,J. und A m r e i c h , J. , Über eine Typhusperitonitis

nach Ruptur eines infolge Infektion mit Typhusbazillen

vereiterten Dermoids 300

Rosenstein, Moritz, Zur Diagnose und Therapie der

Extrauteringravidität . 191

Rübsamen, W., Die operative Behandlung der rektovagi-

nalen Radiumfisteln durch partielle Kranialwärtsverlage-

rung des Afterschließmuskels 307

$\mathrm{S}$ a c h s , E. , Über abdominale Operation hochsitzender Blasen-

zervixfisteln 245

Sperling, Erfolge der Diathermie bei gynäkologischen

Affektionen 309

Stephan, Siegfr. , Indikationsstellung zur Röntgen-

behandlung der Peritoneal- und Genitaltuberkulose . 314 Treber, Hans, Dauerergebnisse der

Aktinotherapie bei

Uterushalskarzinom . 40

Zangemeister, W., Über retroperitoneale Cysten. . . Ill Zweifel, Erwin, Über einen Fall von

akutem Ödem der

Portio bei der Geburt 20

$-\mathrm{VI}-$

Seite

Referate aus geburtshülflich-gynäkologischen Gesellschaften

und Vereinen 76, 335, 380

Geburthülflicli - gynäkologische Mitteilungen aus anderen medizinischen Gesellschaften und Veremen

76, 131, 201, 266, 336, $\quad 390$

Buchbesprechungen 135, 203, 268, 337

Literatur-Verzeichnis 78, 136, 210, 272, 339, 391

Personalien und Tagesnachrichten .... 80,198,276, 340

Druckfehlerberichtígung 214

Berichtigung 396 